\title{
Statistical properties and classification of $N-2$ contingencies in large scale power grids
}

\author{
P. A. Kaplunovich, K. S. Turitsyn \\ Department of Mechanical Engineering \\ Massachusetts Institute of Technology \\ Cambridge, 02139, USA \\ Email: pekap@mit.edu, turitsyn@mit.edu
}

\begin{abstract}
We present the systematic analysis of all dangerous $N-2$ contingencies observed in medium size model of Polish power grid with about 2600 power lines. Each of the dangerous contingencies is composed of two initially tripped lines and one or more lines that overloaded as the result. There are 443 distinct contingencies that do not lead to immediate islanding of the grid. In the scope of the work we analyze the statistics of individual line participation in those contingencies and show that some lines have anomalously high rate of participation in the contingencies. Next, we show that about third of all the contingencies can be associated with the subgrids that are connected to the rest of the grid via small set of power line chains. The contingencies arise when cutting some of those chains results in overload of the others. Simple reduction of power grid corresponding to aggregation of chain components significantly reduces the total number of distinct contingencies. The rest of the contingencies are closely related to a set of almost dangerous $N-1$ contingencies that result in heavy loading of particular lines. Tripping many different additional lines on top of these $N-1$ contingencies results in an overload of one or more lines. We conclude our work by characterization of the joint distributions of power flows through the initiating and overloaded lines and statistical analysis of topological distance between the initially tripped and overloaded lines.
\end{abstract}

\section{INTRODUCTION}

Major power system blackouts are one of the most disastrous catastrophes that can impact the normal life of the country in a most dramatic way. Despite the efforts put in design and control of the power system that ensure its reliable operation the global frequency of major blackouts is increasing with time [1]. Recent blackout in India has left almost $10 \%$ of world population without power supply for several days [2]. Other famous examples include the 2003 easter grid blackout [3] and Arizona-California outage in 2011 [4]

One of the major mechanisms of the blackout formation is the cascading failure where initial failures of individual components result in overloads or other violations of safe operation constraints and lead to either physical failures or protective relay tripping of other system components. This way relatively small initial disturbances can propagate throughout the system in an explosive fashion somewhat similar to spreading of forest fires or infectious diseases [5, 6]. The major difficulty in prevention and even modeling of cascading failures lies in very non-local nature of interactions in power systems. Physical coupling of power grid elements via nearly instant electromagnetic interaction is both a blessing and a curse of power grids. It allows a system to respond easily to minor disturbances and compensate for power flow imbalances by readjusting the power flows on spatially separated components. On the other hand, when the disturbance is large the response of the system may lead to an overloads or even loss of stability of the power grid.

Identification of the events and grid configurations that may lead to a cascading outage is crucially important for prevention and/or mitigation of the damage due to cascading failures. Typically, cascades are triggered by an initial failure of few elements of the power grid. Independent system operators are required to keep the grid protected against the loss of any single power element[7], i.e. they should operate safely in any of the $N$ so-called $N-1$ contingency configurations ${ }^{1}$. Provided that the grid is protected against all $N-1$ contingencies, the actual risks of major cascades depend on the probality of higher order $N-k$ contingencies and the outcomes of the system dynamics after their occurence. One of the major challenges that prevents the proper assessment of cascading failure grids is the computational complexity of $N-k$ contingency selection problem. The total number of contingencies grows exponentially fast as $N^{k}$ and full identification becomes unfeasible even at $k=2-4$ even for moderately sized grids. Recently a number of efficient techniques have been proposed to tackle this problem and identify the most dangerous contingencies using various heuristic algorithms [8-14].

This work builds upon the algorithm described in [13] and is focused on the analysis of the nature of the dangerous $N-2$ contingencies that determine the major risks in the system. We use the largest publicly available model that mimics the structure of Polish power grid. As the number of such contingencies is relatively large (443) we use several statistical

${ }^{1}$ Here and below $N$ denotes the number of lines of the power grid 
approaches to characterize them. Specifically we look for the frequencies of line participation in the contingencies both as part of initiating pair and overloaded lines. Then, we move on to characterization of the relative influence graph topology and provide a classification of the observed contingencies structures. Finally, we analyse the observed correlations in power flows over the overloaded and initially tripped lines.

Although our results do not attempt to improve any specific power grid operation technologies we believe that better understanding of the configurations that may trigger the cascading failures is essential for an improvement of power system reliability. Statistical analysis points out the most important elements of the power grid from the contingency perspective and suggest the optimal ways of improving its reliability. This information can be also used for design of remedial actions for selective set of $N-2$ contingencies where the full analysis is not feasible. Finally, the double contingency influence graph can be used for training of power grid operators and a real-time monitoring of the system health.

In this work we consider only the contingencies associated with tripped power lines, i.e. we don't consider the events where generator or load buses are tripped, although such an extension would be rather straightforward. The double contingency is characterized by a pair of different lines $(x, y)$ each taken from the full set of lines $\mathcal{C}$ in the power grid. We assume that the tripping of these lines happens nearly simultaneously and there is no redispatch of generators between these events. In this case the generation and consumption levels remain the same, and the power flows are determined by the Kirchhoff laws. In this work we use the DC approximation to model the power flows, although the analysis methodology is applicable to more realistic nonlinear models as well.

In order to assess the damage produced by an individual contingency we compare the resulting power flows in the tripped network to the thermal limits of the lines. Whenever at least one of the constraints $z$ becomes violated, we call the contingency dangerous, otherwise it is assumed to be safe. We restrict ourselves to the line thermal constraints as others would require going beyond the DC approximation. To summarize, each dangerous contingency is characterized by a pair of initially tripped lines $(x, y)$ and a set of $\left(z_{1}, \ldots z_{n}\right)$ of lines that become overloaded after the two initiating lines are tripped.

We have used the pruning algorithm proposed in [13] to identify all the dangerous $N-2$ contingencies in an adjusted Polish grid model $^{2}$ from MATPOWER package [15] (see figure 1 for visualization). This model consists of 2631 lines and 2105 buses and the state that we are considering in this study is protected against all $N-1$ contingencies. In other tripping of any line does not lead to islanding or a thermal constraint violation

In our analysis of $N-2$ contingencies we restrict ourselves only to those configuration that remain connected after the tripping of two lines. Double contingencies that immediately

\footnotetext{
${ }^{2}$ We have aggregated the radial branches of the grid into single nodes
}

lead to islanding like the one shown on Fig. 2 are excluded from the set right away. Although these contingencies may lead to power outage if the island does not have enough power generation, we don't consider them in our study as they do not lead to any constraint violation and consequent cascading failure propagation. The total number of $N-2$ contingencies that does not result into an island but leads to at least one constraint violation is 443 . In the forthcoming sections we analyze their structure and statistical properties in details.

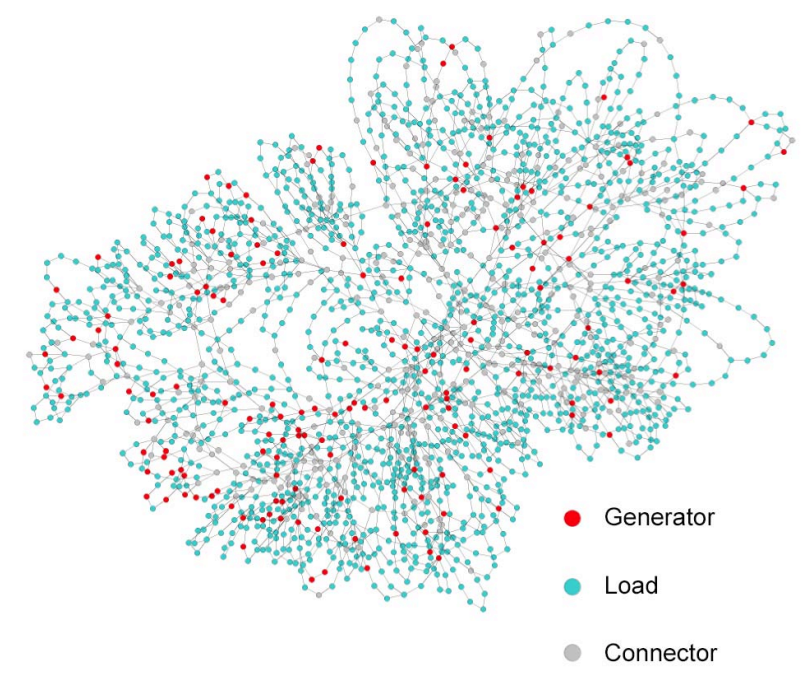

Fig. 1. Physical layout of Polish Grid (positions of the nodes don't correspond to the real grid since geographical data is not presented in the case that has been used

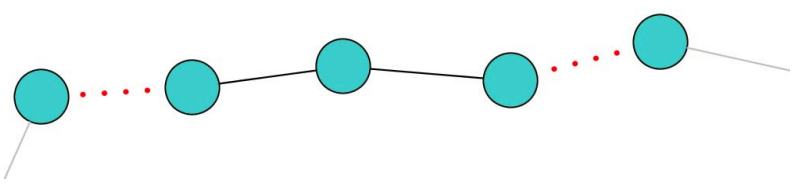

Fig. 2. Example of double contingencies that immediately lead to islanding. Dotted lines represent initiating contingency lines.

\section{GENERAL PROPERTIES OF DANGEROUS CONTINGENCIES}

Our first goal will be to characterize the basic statistical features of the contingency set and its relation to the physical lines in the power grid. There are only 224 power lines that appear as one of the initiating lines. On average 2.75 constraints are violated in a single contingency. However, the set of all constraints that can be violated by at least one double contingency is relatively small: it consists of only 115 unique lines. Different lines appear in contingency set with different frequency, which can be easily seen from the figures 3 and 4 where we plot the distribution of line participation frequency in the sets of initiating lines and violated constraints correspondingly. 


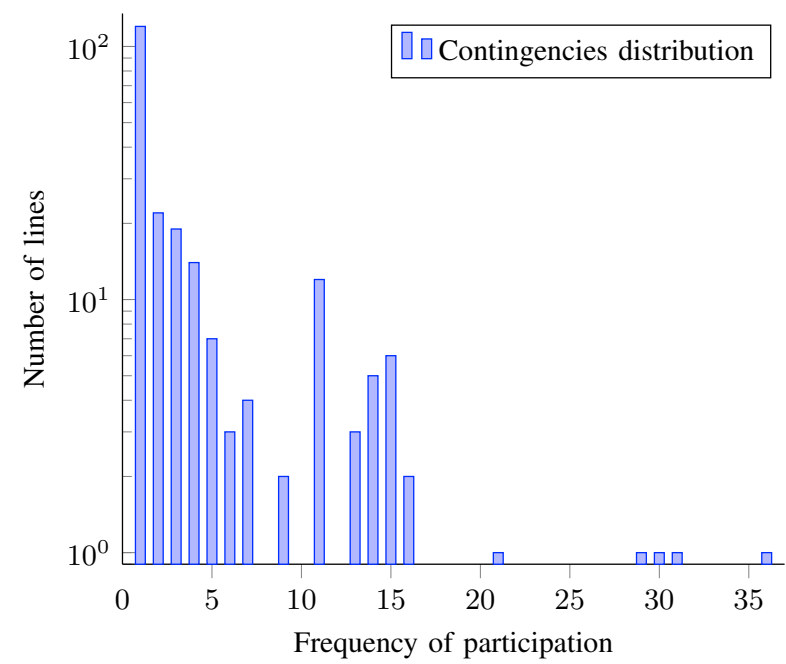

Fig. 3. Contingencies distribution (224 unique participants)

Distribution in the figure 3 shows that although typical lines enter only few contingencies, there are 5 lines that participate in at least 20 different double contingencies. These lines are likely responsible for the major risks in the power grid. If the initiating trippings are separated in time, the initial trips makes the grid vulnerable to more than 20 additional line trips. The quantitative assessment of these lines contributions to the overall risk can not be done without a specification of the tripping probabilities. However, under reasonable assumptions of comparable probabilities of each line trips one may conclude that the reliability of the power grid is highly sensitive to the tripping probability of those lines. From a more positive perspective, decrease of these lines tripping probability is probably one of the most cost-efficient ways of improving the system reliability.

In figure 4 we show the corresponding distribution of the number of times a given line has been overloaded by one of the contingencies. This distribution has even more pronounced tails, with about 20 lines becoming overloaded in more than 20 contingencies and 5 of them being overloaded in more than 50. Most of these lines get close to their limit after one or several $N-1$ contingency, and can easily get above the limits whenever an additional second line is tripped. As in the case with initiating lines, some reinforcement or simple deloading of a few of the most frequently overloaded lines can lead to dramatic improvements in reliability of the system, or at least decrease the number of dangerous $N-2$ contingencies. For instance, by increasing the thermal limits of the line that participated 68 times one can decrease the number of dangerous contingencies by 56 , which is $13 \%$ of all 443 contingencies (the number of contingencies is not decreased by 68 , because there is also other violations in some cases when the line was overloaded).

Next, in order to understand better the structure of the double contingencies we have analyzed more closely the

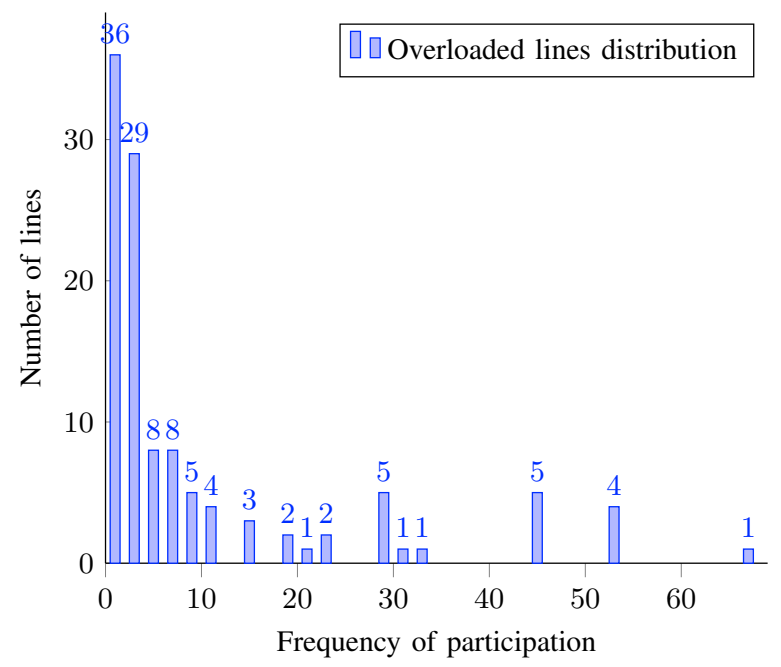

Fig. 4. Overloaded lines distribution (115 unique participants)

relative influence of different lines on each other. The first natural question is what lines do appear together as initiating pairs. In order to answer this quesiton we visualize the set of pair contingencies as a graph where the nodes represent the initiating lines, and edges represent the individual double contingencies. In other words, every contingency initiated by a pair $(x, y)$ is represented by two vertices $x$ and $y$ and and edge connecting these vertices. The resulting graph is represented in the figure 5. As one can see the graph has multiple disconnected components with very different structure. In the following sections we attempt to understand the nature of these components and interpret the results in terms of structures on actual power grid.

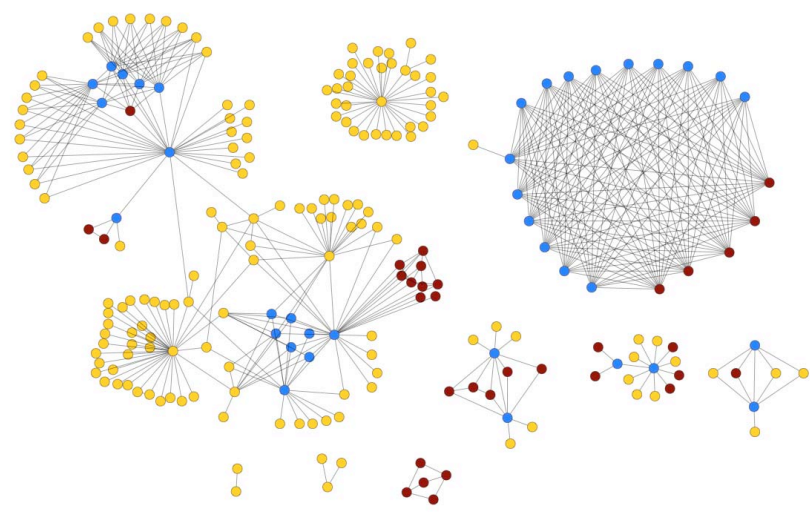

Fig. 5. Contingecies graph. Each contingency is represented by two vertices connected by line. Red nodes represent initiating lines that participate in only "islands" contingency scenario, yellow nodes are for initiating lines that never participate in such contingencies (and hence are never connected to red ones), blue nodes represent initiating lines that can be observed in both scenarios. Different scenarios are disscussed in section 3

One of the most striking features of the graph is a highly variable sparsity structure. Although most islands appear to be 
rather sparse with only few edges per node, the cluster in the upper right part of the graph is characterized by the very high node degree. Inspection of this cluster reveals that in this and several other situations the high degree of interconnection is related to the topology of actual power lines that form these clusters. High level of interconnections typically occur when the power lines are organized in "chains": one-dimensional segments of serially connected lines as shown in figure 6 . Tripping of any line on such a segment leads to nearly the same effect: prevents the power to flow between its ends. Whenever two of such chains of length $n_{1}$ and $n_{2}$ participate in a contingency, there are $n_{1} n_{2}$ possible ways of cutting both chains and causing the same or similar effect. So, the two chains produce a total of $n_{1} n_{2}$ contingencies. Although this statement is not rigorous and should not necessarily hold for any chains, it was true for all chains participating in contingencies in the grid considered in this paper. This observation also suggests that a number of a contingencies is not really an appropriate metric of a grid reliability as this number will depend on how the level of granularity of the grid model.

In order to account for this effect and reduce the doublecounting of similar contingencies we have performed an additional virtual reduction of the grid where the onedimensional chains of lines were "collapsed" into the single elements as shown in the figure 6. Algorithmically this procedure corresponds to relabeling of the lines, in a way that physically different lines belonging to the same chain are labeled with the same chain identifier. Thus the power flow calculations are not affected by reduction - only data representation is. Such virtual reduction helps to capture the essence of the phenomena without counting the same effect multiple times.

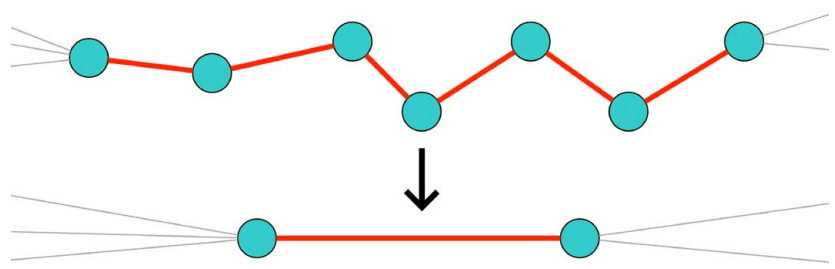

Fig. 6. Illustration of reduction procedure. Figure shows how chain of lines is substituted by one line

After the reduction we observed a significant decrease in the total number of contingencies and as well as in the number of initiating and overloaded lines participating in these contingencies. To check whether the reduction has affected significantly the participation frequency distribution we have plotted the updated distribution in figures 7 and 8. Although the total number of lines participating in contingencies has decreased significantly, and both typical and extreme participation frequencies are lower after the reduction, we see that the qualitative shape of the distribution is still the same. Most of the lines participate in only few contingencies, however there are a few that participate in many of them.

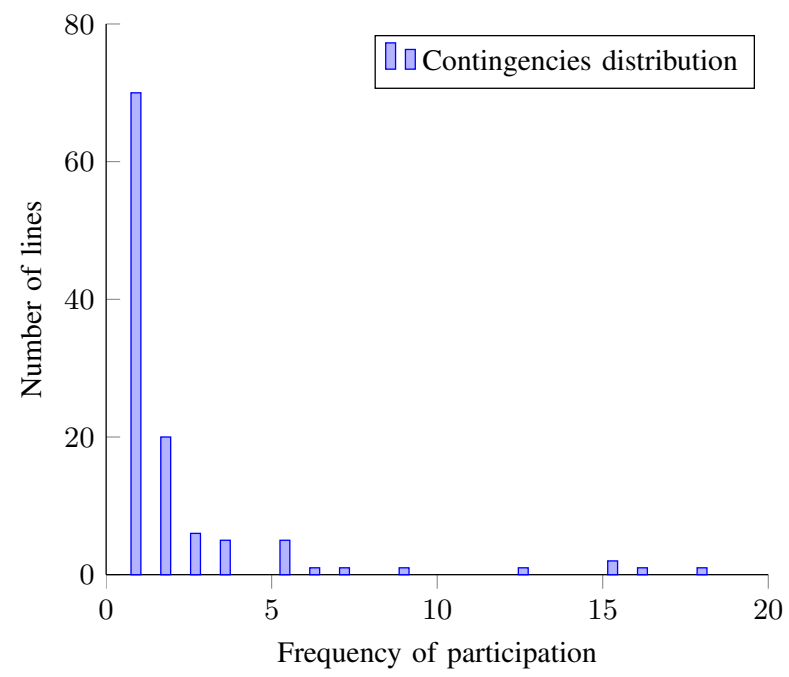

Fig. 7. Contingencies distribution after grid reduction (114 unique chains)

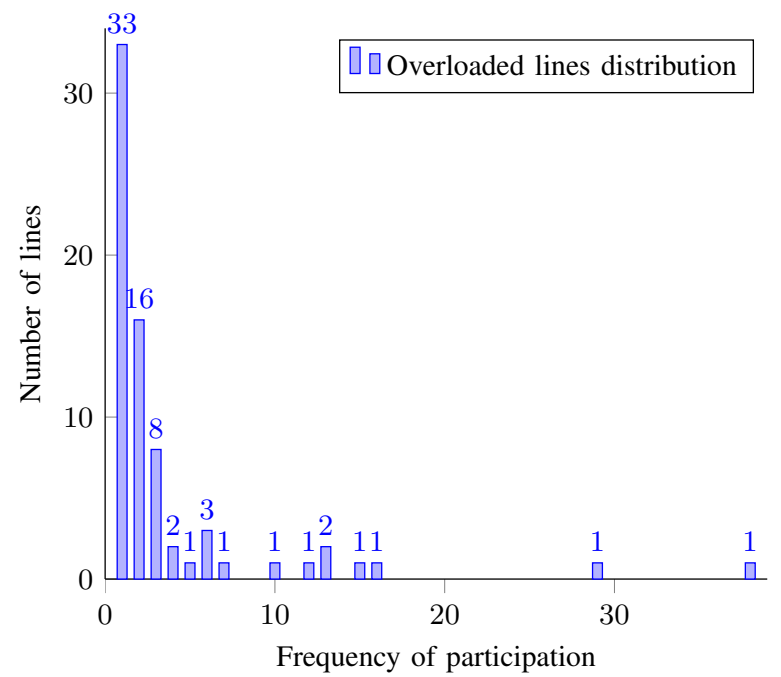

Fig. 8. Overloaded lines distribution after grid reduction (72 unique chains)

The effect of the reduction procedure in the contingency graph and the resulting new graph are presented in Fig. 9 and Fig. 10 respectively. As expected, the most interconnected regions in the updated graph are gone and the upper-right component in Fig. 5 with multiple connections is collapsed in only three-node island in the reduced contingency graph. The lines from the right tail of the distribution in 7 are seen as the "hubs" in Fig. 10. These graphs also reveal an additional information and suggest how removal of some of the most dangerous lines will reduce the total number of the lines participating in the double contingencies thus reducing the overall risks in the system. Indeed, there are a lot of star- 


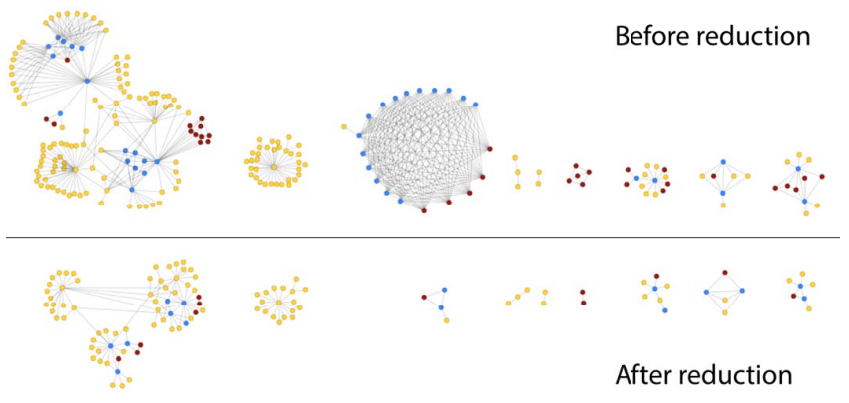

Fig. 9. Transformation of contingency graph after grid reduction. Corresponding parts are verticaly alignied

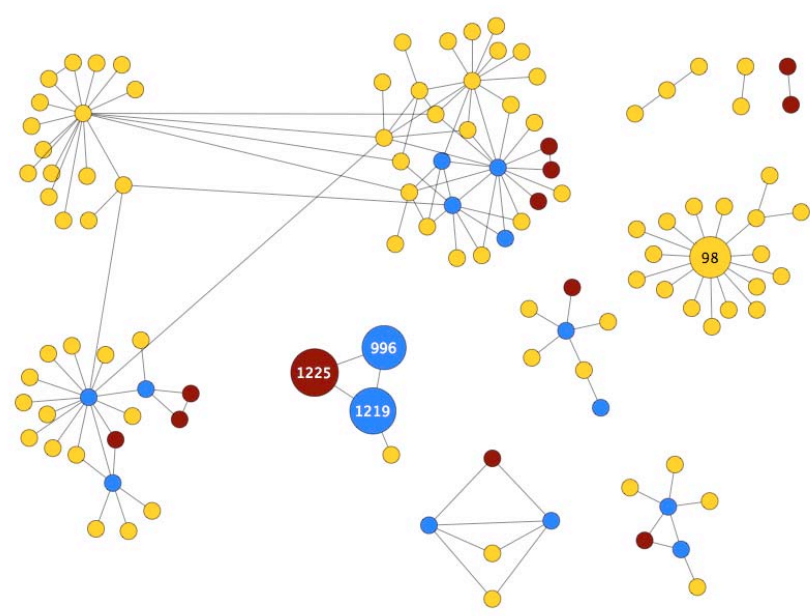

Fig. 10. Contingency graph after reduction

shaped subgraphs in 10 that would completely disappear if their center nodes were protected and their tripping probability was reduced.

The contingency graph presented in figures 5 and 10 visualizes the information about participation of individual lines as initiators of the contingency. However, it does not provide any information about what lines are overloaded as the result of contingencies. In order to address this question we have processed the contingency set and presented its structure in a new type of the graph that we call influence graph presented in Fig. 11. In this graph we put together both the lines that have initiated the contingency and the lines that became overloaded as a part of it. Some of the lines may participate both as initiating and overloaded lines in different contingencies. To assist visualization we separate the participating lines in three columns. Left column corresponds to the lines that enter in the contingency set only as initiating lines. The most right column corresponds to the lines that may get overloaded but never initiate a contingency. Finally, in the middle column we have those lines that can be overloaded in some contingencies and may initiate some others. As one can see every node in the middle and right columns has at least two edges connected to it, indicating that they are overloaded

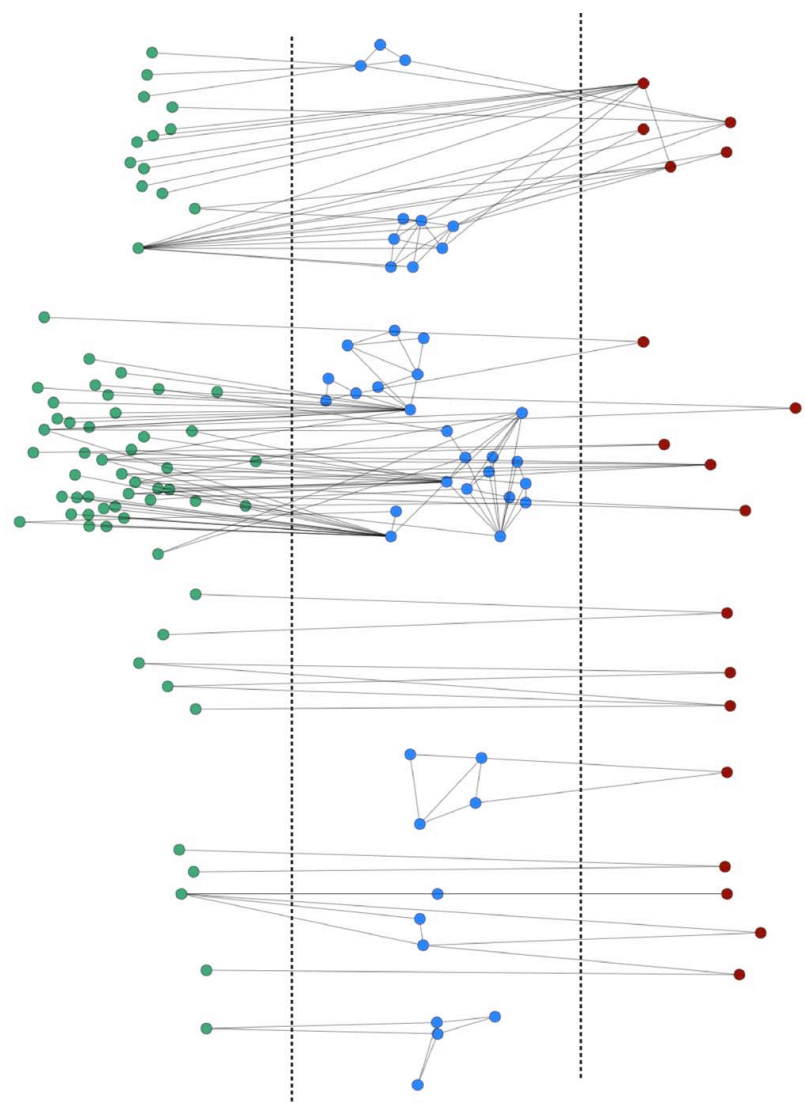

Fig. 11. Influence graph after reduction of the grid. Green vertices represent lines that can only be initiators of dangerous contingencies; Red nodes represent lines that never initiate contingencies but can become overloaded by some of them; Blue nodes represent lines that can be overloaded by dangerous contingencies as well as initiate them

by double contingencies. For the sake of visual appeal we use an undirected graph for visualization, so some information about the composition of contingencies is not presented.

Remarkably, the middle column is more populated than the right one, which suggests that for many contingencies the tripping of the overloaded line may trigger other overloads and initiate the full-scale cascade of failures. Note, that this statement is only an educated guess as the accurate account of effect of third line tripping requires full-scale recalculation of the power flows in an updated $N-3$ network. Although there is not enough information in the double contingency set to predict what will happen if one or few of the overloaded lines will trip, one may assume that the overall stress on the system will only increase and more overloaded lines will appear. If this conjecture is accepted, one can use the information provided in the influence graph for rough estimates of the danger of the contingencies and use it for contingency ranking or remedial action development procedures.

In order to better understand the structure of the double contingencies in the following section we analyze several of the components of the contingency and influence graphs and discuss what are the typical configurations that result in 
dangerous double contingencies.

\section{StruCture of DANGEROUS CONTINGENCIES}

In our system we have observed two major classes of double contingencies. The first class corresponds to configurations where a subgrid is connected to the rest of the grid via a small number of power lines that form a so called graph cut. One or more of these lines can get overloaded if other lines in the same graph cut are tripped. Second important class of contingencies corresponds to nearly-dangerous $N-1$ contingencies that require only a small variation due to second line tripping to become really dangerous. In the following paragraphs we analyze these scenarios in more details.

The "island"-class contingencies constitute about $31 \%$ of all the dangerous contingencies before the reduction. They appear whenever a some subgrid of the system is connected to the rest of the grid via a set of lines (that constitute a graph cut) with relatively small overall power flow limit. Whenever one or two lines from the cut are tripped, the total power that was flowing through the cut is redistributed among the remaining lines. This can lead to an overload of the remaining lines in the cut. As long as there are many ways to draw cuts in the power grid graph, we assume that a given contingency is of "island" type only if all the remaining lines in the cut are overloaded after the initial tripping events. The likely outcome of such a contingency is the islanding of the subgrid after the overloaded lines are tripped. Depending on the distribution of load and generation within this island the cascade might propagate further or remain contained in this island.

In figures 5 and 10 we show the lines that participate in these types of contingencies in red and blue colors. Red indicates that the line participate only in "island" type of contingencies, and blue is used for lines that can participate in other types of contingencies as well. Yellow lines do not participate in "island" type of contingencies at all.

In figure 12 we show an example of such an islanding contingency illustrating in the same figure both the physical layout of the corresponding island, its representation in the contingency graph before and after reduction, and, finally, its representation in the influence graph. As one can see from the section 4 of this figure there are two chains of power lines interfacing a small subgrid with the power system. Cutting any two chains in any of their lines results in an overload on the third chain. One can note, that the relative large size of chains results in a large number of contingencies before the graph reduction. One can also see from the influence graph that it does not matter which of the chains are cut as in the contingency - cutting any two of them will result in an overload of the third chain.

The second class of contingencies is not related to the weakly connected islands and does not have any specific physical layout signatures. They arise due to the large number of $N-1$ contingencies that are close to the margin of being dangerous, so that whenever such a contingency occurs, one of the lines in the power grid becomes almost overloaded. Tripping of other line in the grid results in additional power
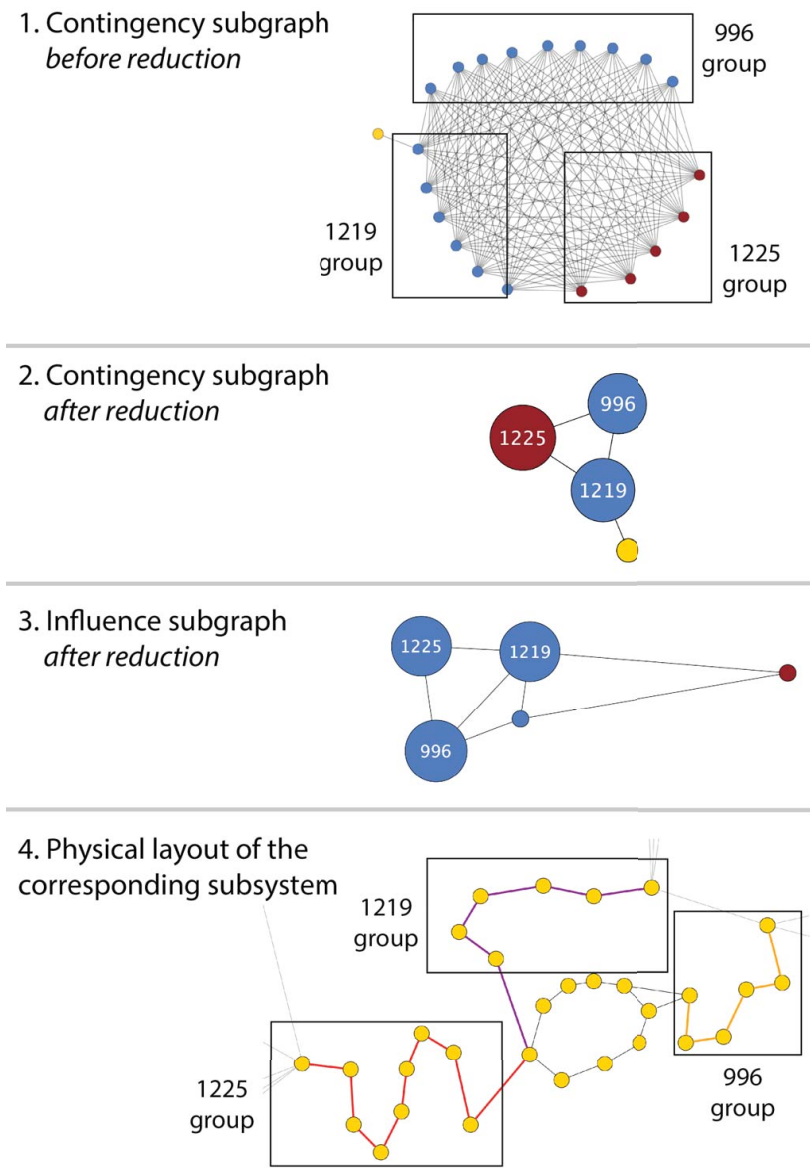

Fig. 12. Example of "island" type of contingency

flow readjustment and may act as a "last straw" pushing the nearly overloaded line over the limit. These types of contingencies constitute a majority of all observed in our numerical experiment. This is not surprising given the fact that the optimal power flow solutions are often found with $N-1$ contingency security condition imposed as a constraint [16] and the long-term evolution of the grid is also driven by the $N-1$ security condition [17]. The solution naturally lies on the boundary of the feasible region thus resulting in near overload of some of the lines. This results in a system exposed to a large number of $N-2$ contingencies. More sophisticated schemes, based on chance constrainted optimization may be required to find the solutions that reduce the overall risks associated with $N-2$ contingencies.

An example of this type of contingency is shown in Fig. 13. The physical layout of the power grid with the lines involved in the contingencies is not shown because the large number of lines that can participate in this subset of contingencies are scattered all over the power grid. The $N-1$ contingency associated with tripping of the central line \#98 in the contingency graph results in high loading of the line \#377. The flow through line \#377 reaches almost $93 \%$ of the line capacity. Any of the lines connected to the central 


\section{Contingency subgraph before reduction}

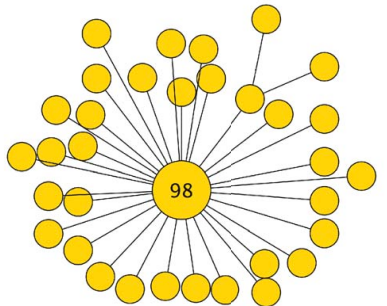

2. Contingency subgraph after reduction

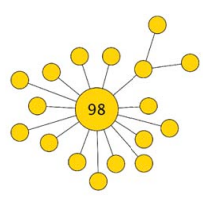

3. Influence subgraph after reduction

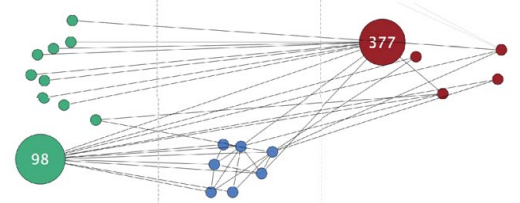

Fig. 13. Example of $N-1$ contingency that is close to margin of being dangerous

one in the contingency graph produces an additional power flowing through the line \#377 leading to the overload.

The non-local nature of the most double contingencies can be illustrated by looking at the distribution of graphical distances between the initiating contingencies and the overloaded lines. This distribution is presented in Fig. 14. As one can see most of the initiating lines are separated from one another by 5 or more nodes in the original power grid with the largest separation being as high as 12 . The distribution of distances between the initiating and overloaded lines is more narrow, however this effect may be attributed to the algorithm of distance calculation, that used the distance between the closest among the set of initially tripped and overloaded lines. In any case, this distribution highlights the nonlocal nature of the contingencies and indicates serious flaws of the topological models based on the nearest neighbor interaction (see also [18] for the discussion of this point). In the future we plan to extend this study to other more appropriate metrics, like electric distance [19].

All the contingencies that we have observed in our study can be explained by these two mechanisms. This characterization of dangerous contingencies may be used as a basis for heuristic contingency selection algorithms in situations where direct non-heuristic approaches are not available or computationally prohibitive. Although the model that we used in our study was based on linear DC power flow equations, the qualitative nature of the overloads is universal and not reliant on any approximations. So we believe that the same type of contingencies will be observed in more realistic AC type models.

In the last results section we provide additional information

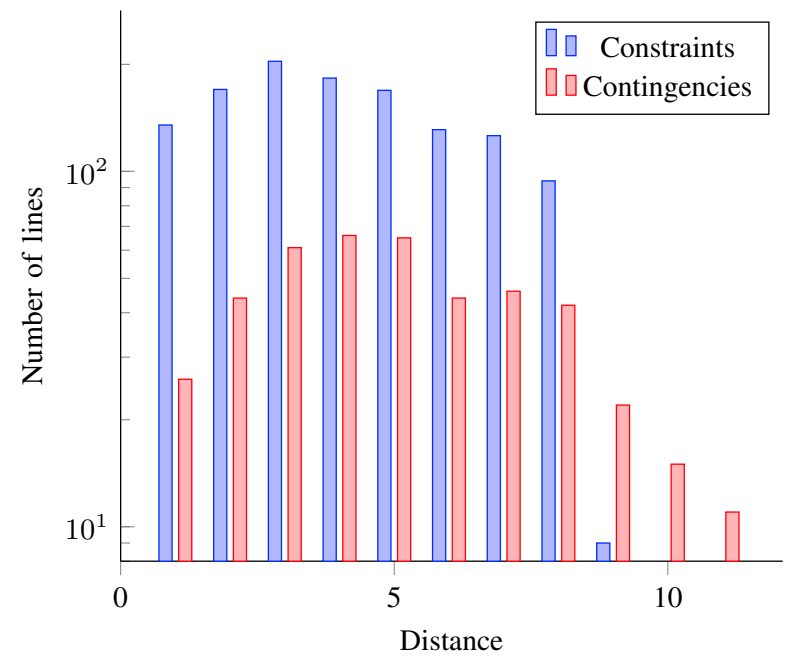

Fig. 14. Topological distances distribution (Blue - distribution of average topological distances between overloaded lines and corresponding initiating lines. Red - distribution of distances between initiating lines

about the power flows through the lines that participate in contingencies either as initiating or overloaded lines.

\section{POWER FLOWS IN DANGEROUS CONTINGENCIES}

In order to better understand the potential impact of the contingencies on the grid we have done preliminary assessment of the power flows through the overloaded lines. We visualize the data on the power flows using the scatter plot presented in Fig. 15. The two axes of this plot represent the power flowing through a given line before and after the contingency and the limit of the power line flow as defined in the model. The blue points correspond to the power flows before the contingency and all lie in the safe region where the limit is larger than the flow. The configurations after the contingency are shown in red an all lie in the lower-right region of the plot where flow exceeds the limit. We also show the values of the power flows averaged over all the lines in the grid. There are several interesting conclusions that one can draw from this plot.

First, one can see that in most of the cases the overloaded lines barely exceed their limits. This is consistent with the nature of the most contingencies that are composed out of nearly dangerous $N-1$ contingencies reinforced by a small push from another tripped line. Most of these contingencies can be avoided if more restrictive conditions for the $N-1$ security are used when choosing the optimal power flow solution.

Second, we can see that most of the lines that get overloaded are operated away from their limits, most at half of their capacity. This is a rather unexpected result which suggests that the overloading effect is mostly due to the readjustment of the flow after the contingencies. In other words it suggests that the most vulnerable lines are not the ones that operate close to their limits, but the ones that are 
the most sensitive to the tripping of others. Alternatively one may argue, that in the optimal power flow analysis the most restrictive constraints are those related to $N-1$ contingency security. On the other hand by looking at the average levels of power flows and power flow limits we see that most of the lines that get overloaded have higher than average flow but lower than average power limit. Some reinformcement or artificial deloading of this line may therefore reduce most of the $N-2$ contingencies.

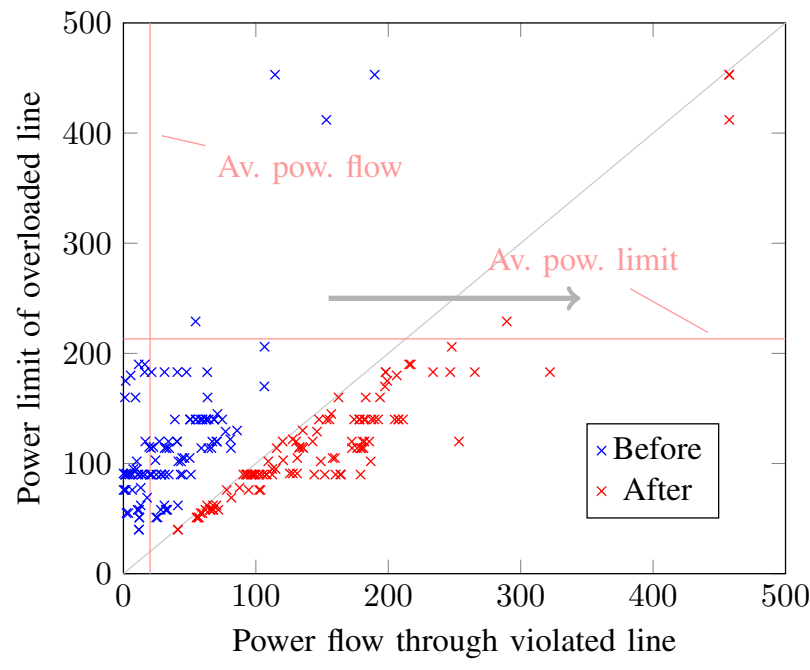

Fig. 15. Scatter plot of power limit versus power flow before and after contingencies through overloaded lines

In order to understand which lines cause the most dangerous contingencies we have looked at the joint distribution of total power flows over the initially tripped lines and the mean change of power flow over the lines that get overloaded as a result of contingency. The corresponding results are presented in Fig. 16. As one can see most of the contingencies are induced by tripping of some major lines in the grid, the typical flows over these lines exceeds the average power flow levels in the grid. This can be explained by the strong effect of tripping major lines in the grid. Trippings of major lines produces stronger effect all over the grid and has higher chances of resulting in a violated constraint. One can also see well-pronounced clusters of contingencies arranged in the lines. These clusters correspond to "island" type contingencies where the the flow from initiating lines gets entirely redistributed to the the overloaded lines. Depending on which of the line in the chain was tripped initially different amount of flow will go through the overloaded lines.

\section{Summary AND CONCLUSIONS}

We have presented a thorough analysis of all dangerous $N-2$ contingencies observed in the medium size model of Polish power grid with about 2000 buses. The power flow model was based on the DC approximation and only line trip contingencies and thermal power constraints were

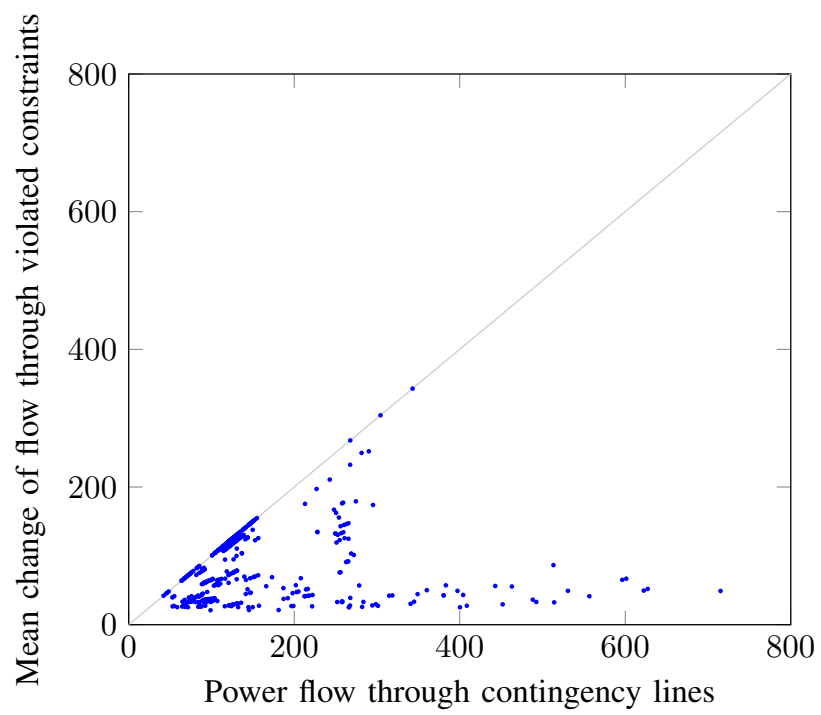

Fig. 16. Scatter plot of sum of power flows through contingencies vs. mean change of power flow through constraint (sum of power flows through violated constrains divided by their number).

considered. We have identified several contingency classes and characterized them via a number of statistical techniques.

The most common type of $N-2$ contingency appears when a certain subgrid is connected to the rest of the grid with only 2 power lines. Whenever these two lines trip the grid becomes islanded. These contingencies have purely topological nature and were not considered in this work. The number of such contingencies is combinatorially large, there are several thousand of them in the Polish Grid model.

Second type of contingencies is also characterized by grid islanding, but in contrary to the previous type in this case the islands are connected to the rest of the grid with more than two power line chains. Whenever two of those lines are cut, the rest can become overloaded. These sort of contingencies constitute about $31 \%$ of the total dangerous contingency count. However, their ratio can be significantly reduced if the long chains of power lines are reduced to a single line in contingency enumeration procedure. The likely outcome of these contingencies is islanding of the grid which can only be prevented by reinforcing of the overloaded lines or reducing tripping probability of the contingency initiating lines.

Finally, the last and by far the most common type of contingencies appears from the nearly dangerous $N-1$ contingencies. Whenever a particular $N-1$ contingency results in high loading of one or more power lines, tripping of many additional lines can lead to overload. In this case this single $N-1$ contingency has multiple $N-2$ counterparts, as many additional lines can lead to overload. Many of these additional lines participating in the $N-2$ contingency are located far away from corresponding original $N-1$ contingency participants. The resulting level of overload is very close to the actual limit. The number of such contingencies can be reduced dramatically by enforcing more 
strict $N-1$ security constraints in Security Constrained Optimal Power Flow procedure.

We have observed highly nonuniform distribution of line participation frequencies in the $N-2$ contingencies set. Only about $10 \%$ of the total number of lines participate in at least one $N-2$ contingencies. And those that do typically participate in only 1 or 2 distinct contingencies. However, there are $5-10$ lines (or about $0.2-0.5 \%$ ) that participate in anomalously high number of contingencies, up to $15-20$ for some lines. Reducing tripping probabilities of these lines can have dramatic effects on the overall reliability of the grid. Similarly, there are only 72 unique chains of power lines that can get overload as a result of $N-2$ contingency. Of those 72 the majority are vulnerable to only $1-3$ distinct contingencies, but some get overloaded in more than 20 configurations. Upgrade of those lines or imposal of tighter constraints on their power is likely the most cost-efficient way of increasing the power grid reliability.

We have also observed that many of the lines participate in the contingency set both as the initiating lines in some contingencies and overloaded lines in others. The contingencies where those lines are overloaded are the natural candidates for events triggering the large scale cascades in power grid. If one those lines gets tripped as a result of the overload the resulting $N-3$ contingency will produce more overloads in the grid. If reinforcement of those lines is not possible, fast remedial action plan can help protect the grid against the development of full scale cascade.

We believe that the analysis carried out in this work can help system operators and the power system academic community to develop novel approaches to grid reliability improvement. The most straightforward application of our results is to use these approaches to identify the lines that participate most frequently in $N-2$ contingencies and find the most appropriate ways of reducing the risks associated with those contingencies. This could be accomplished either by physical upgrades of the lines, or introduction of additional security motivated constraints in OPF procedures. However the results may also be used in more sophisticated and indirect ways. The statistical properties of the $N-2$ contingencies may be used to bias the distribution of starting configurations of cascade simulation software based on more realistic models of power grid dynamics. This way faster convergence of the Monte Carlo based algorithms may be achieved [20]. It may also help improve various heuristics that were proposed for contingency selection problems.

Finally, we want to emphasize that there are a lot of questions that still need to be resolved before the full understanding of dangerous $N-2$ contingencies nature is achieved. Some specific questions that we would like to address in the nearest future include but are not limited to the following. Current analysis was carried out for a single operating point of the grid. It is not clear how robust are the results to variations of the operating point. Different operating points may have different participants of the contingency sets. The most important lines have to be identified by averaging over the ensemble of possible states of the systems, and the resulting set of lines may be very different from the ones identified in this study. To have some quantitative suggestions on the risk reduction actions it is also important to introduce the probability measures that characterize the frequency of initiating line trips and some ways of assessing the damage done by particular contingencies. The latter task can be naturally accomplished with the help of simple cascade simulators proposed by a number of groups in recent years [21-23].

\section{ACKNOWLEDGEMENTS}

We thank NSF, MIT/SkTech initiative, and Russian Ministry of Education for their support.

\section{REFERENCES}

[1] P. Hines, J. Apt, and S. Talukdar, "Large blackouts in north america: historical trends and policy implications," Energy Policy, vol. 37, no. 12, pp. 5249-5259, 2009.

[2] D. Goswami, "A 10-step plan to end india's blackout," Renewable Energy Focus, vol. 13, no. 5, pp. 26-28, 2012.

[3] B Liscouski and W Elliot, "Final report on the august 14, 2003 blackout in the united states and canada: causes and recommendations," A report to US Department of Energy, vol. 40, 2004.

[4] F. S. Report, "Arizona southern california outage on september 8 2011," 2012.

[5] D. J. Watts, "A simple model of global cascades on random networks," Proceedings of the National Academy of Sciences, vol. 99, no. 9, pp. 5766-5771, 2002.

[6] S. V. Buldyrev, R. Parshani, G. Paul, H. E. Stanley, and S. Havlin, "Catastrophic cascade of failures in interdependent networks.," Nature, vol. 464, no. 7291, pp. 1025-8, Apr. 2010.

[7] NERC, "Standard FAC-011-2 - System Operating Limits Methodology for the Operations Horizon," 2009.

[8] Q. Chen, S. Member, and J. J. D. Mccalley, "Identifying High Risk N-k Contingencies for Online Security Assessment," IEEE Transactions on Power Systems, vol. 20, no. 2, pp. 823-834, May 2005.

[9] C. Davis and T. Overbye, "Linear Analysis of Multiple Outage Interaction," in 2009 42nd Hawaii International Conference on System Sciences, IEEE, 2009, pp. 1-8.

[10] C. M. Davis and T. J. Overbye, "Multiple Element Contingency Screening," IEEE Transactions on Power Systems, vol. 26, no. 3, pp. 1294-1301, Aug. 2011.

[11] M. J. Eppstein and P. D. Hines, "A "random chemistry" algorithm for identifying collections of multiple contingencies that initiate cascading failure," Power Systems, IEEE Transactions on, vol. 27, no. 3, pp. 1698-1705, 2012. 
[12] P. D. Hines, I. Dobson, E. Cotilla-Sanchez, and M. Eppstein, " "dual graph" and "random chemistry" methods for cascading failure analysis," in Hawaii International Conference on System Sciences, 2013.

[13] K. Turitsyn and P. Kaplunovich, "Fast algorithm for n-2 contingency problem," in 2013 46th Hawaii International Conference on System Sciences (HICSS), 2013, pp. 2161-2166.

[14] P. Kaplunovich and K. Turitsyn, "Fast selection of n-2 contingencies for online security assessment," in IEEE Power Engineering Society general meeting, arXiv preprint arXiv:1303.3938, 2013.

[15] R. D. Zimmerman, C. E. Murillo-Sanchez, and R. J. Thomas, MATPOWER: Steady-State Operations, Planning, and Analysis Tools for Power Systems Research and Education, 2011.

[16] D. Gan, R. J. Thomas, and R. D. Zimmerman, "Stability-constrained optimal power flow," Power Systems, IEEE Transactions on, vol. 15, no. 2, pp. 535-540, 2000.

[17] H. Ren, I. Dobson, and B. A. Carreras, "Long-term effect of the n-1 criterion on cascading line outages in an evolving power transmission grid," Power Systems, IEEE Transactions on, vol. 23, no. 3, pp. 1217-1225, 2008.

[18] P. Hines, E. Cotilla-Sanchez, and S. Blumsack, "Do topological models provide good information about electricity infrastructure vulnerability?," Chaos: An Interdisciplinary Journal of Nonlinear Science, vol. 20, no. 3, pp. 033 122-033 122, 2010.

[19] E. Cotilla-Sanchez, P. D. Hines, C. Barrows, and S. Blumsack, "Comparing the topological and electrical structure of the north american electric power infrastructure," IEEE Systems Journal, vol. 6, pp. 616-626, 2012.

[20] Q. Chen and L. Mili, Risk-based composite power system vulnerability evaluation to cascading failures using importance sampling, 2011.

[21] D. P. Nedic, I. Dobson, D. S. Kirschen, B. A. Carreras, and V. E. Lynch, "Criticality in a cascading failure blackout model," International Journal of Electrical Power \& Energy Systems, vol. 28, no. 9, pp. 627-633, 2006.

[22] C. Parmer, E. Cotilla-Sanchez, H. K. Thornquist, and P. D. Hines, "Developing a dynamic model of cascading failure for high performance computing using trilinos," Proceedings of the first international workshop on High performance computing, networking and analytics for the power grid - HiPCNA-PG '11, p. 25, 2011.

[23] M. Chertkov, R. Pfitzner, and K. Turitsyn, "Controlled tripping of overheated lines mitigates power outages," Los Alamos National Laboratory (LANL), Tech. Rep., 2011. 